\title{
HYDROCARBON INSECTICIDES: THEIR RISKS FOR ENVIRONMENT AND HUMAN HEALTH
} \author{
By \\ MAMDOUH M. EL-BAHNASAWY' ${ }^{1}$, AMINA EL-HOSINI MOHAMMAD ${ }^{1}$ \\ AND TOSSON A. MORSY ${ }^{3}$ \\ Military Medical Acadeny ${ }^{1}$ and Department of Parasitology, Faculty of Medicine, Ain \\ Shams University, Cairo $11566^{2}$, Egypt
}

\begin{abstract}
Insecticides are used to control diseases spread by arthropods, but theys vary greatly in toxicity. Toxicity depends on the chemical and physical properties of a substance, and may be defined as the quality of being poisonous or harmful to animals or plants. Poisons have many different modes of action, but in general cause biochemical changes which interfere with normal body functions. Toxicity can be either acute or chronic. Acute toxicity is the ability of a substance to cause harmful effects which develop rapidly following absorption, i.e. a few hours or a day. Chronic toxicity is the ability of a substance to cause adverse health effects resulting from long-term exposure to a substance. There is a great range in the toxicity of insecticides to humans. The relative hazard of an insecticide is dependent upon the toxicity of the pesticide, the dose received and the length of time exposed. A hazard can be defined as a source of danger.

The great majority of insecticides are poisonous to man and his beneficial insects and animals and are carcinogenic agents particularly, the halogenated hydrocarbons containing benzene ring.
\end{abstract}

Key words: Halogenated hydrocarbons, environmental pollution, Human health

\section{Introduction}

Hydrocarbons are organic substances that contain carbon and hydrogen. They are generally liquid at room temperature. All the petroleum distillates (e.g., kerosene, gasoline, mineral seal oils, \& naphtha) are hydrocarbons; however, not all the hydrocarbons are petroleum distillates. The turpentine, for example, is a hydrocarbon made from pine oil. Hydrocarbons are often mixed with agents that have systemic toxicity such as camphor, aniline dyes, heavy metals, and pesticides (Lewander and Aleguas, 2007).

Ingestion of large quantities of hydrocarbons alone by children is unusual because hydrocarbons are foul-tasting. Poisoning in young children typically results from an exploratory occurrence that can be prevented by safe packaging and storage. In contrast, in teenagers and adults, aspiration may occur during intentional behaviors e.g., during inhalant abuse, or when attempting to siphon gasoline (Bronstein et al, 2007).

Epidemiology: The hydrocarbon ingestion accounts for two to three percent of non- pharmaceutical exposures in children less than six years of age reported to US poison control centers (Bronstein et al, 2008). Although rare, hydrocarbon aspiration may cause death secondary to respiratory failure. The gasoline, chlorofluorocarbon propellants, motor oils, mineral spirits, lighter fluid/ naphtha, and lamp oil are the most commonly ingested substances. In young children, the ingestion typically occurs as a result of exploratory behavior. Frequently, the hydrocarbon is unsecured or improperly stored in a drinking container (e.g., soda bottle). Toxicity in adolescents often arises from inhalant abuse of hydrocarbons (WHO, 2010).

Product safety: Since 2001, US consumer product safety commission has required child-resistant packaging for products that have low viscosity and contain $\geq 10 \%$ hydrocarbon by weight (Barone, 2002). In 1997, the European Union attempted to minimize lamp oil aspiration by setting standards for viscosity and surface tension, the main determinants of aspiration risk. A study in 
2005 showed no effect on number or severity of hydrocarbon aspiration in years since the directive (Van Gorcum et al, 2009).

Classes of Hydrocarbons: Four structural classes of hydrocarbons are Aromatic hydrocarbons aliphatic hydrocarbons Halogenated hydrocarbons Terpene hydrocarbons

Aromatic hydrocarbons are cyclic compounds containing a benzene ring (e.g., benzene, toluene, and xylene) used primarily in solvents, glues, nail polishes, paints, and paint removers. The halogenated ones are fluorinated, chlorinated, or brominated (as the methylene chloride chloroform, carbon tetrachloride, trichloroethylene tetrachloroethylene). Systemic toxicity also occurs after ingestion of compounds that combine aliphatic hydrocarbons with toxic additives e.g., organophosphates, heavy metals, camphor (Khambay and Jewess, 2004).

The terpenes include turpentine and pine oil. Aliphatic hydrocarbons are petroleum distillates such as gasoline, kerosene, and naphtha. They are found in the furniture polishes, the lamp oil, and lighter fluid (Lewander and Aleguas, 2007).

Hydrocarbon Toxicity: Hydrocarbons can be classified according to the potential for toxicity: Low toxicity (unless complicated by gross aspiration). These include asphalt, tars, mineral oil, petroleum jelly, motor oil, and axle grease. Aspiration hazard and clinical effects are typically limited to direct pulmonary risk, as the turpentine, gasoline, kerosene, mineral seal oil (furniture polish), charcoal and cigarette lighter fluids, and mineral spirits. The systemic toxicity of halogenated and aromatic hydrocarbons is absorbed readily through the gastrointestinal and/or respire-tory systems. Systemic effects include cardiac arrhythmias secondary to myocardial sensitization \& CNS depression.

Determinants of the pulmonary aspiration: The aspiration hazard of the hydrocarbons is determined by three properties: Volatility The ability to vaporize or to exist in a gaseous form the greater the volatility, the greater the risk of pulmonary absorption and CNS depression surface tension, adherence of molecules along a liquid surface. Lower surface tension allows compounds to spread or "creep" over a larger area. Viscosity - The resistance to flow through an orifice or the tendency of a compound to resist "stirring". Lower viscosity facilitates deeper penetration into the tracheobronchial tree (Lewander and Aleguas, 2007).

The aspiration hazard of hydrocarbons is inversely related to viscosity and surface tension and directly related to volatility. So, hydrocarbons with decreased viscosity, low surface tension, and high volatility are more likely to be aspirated and cause pulmonary injury. Low viscosity permits greater penetration into distal airways while the low surface tension facilitates spread over a larger area, as an example, simple petroleum distillates (kerosene, gasoline, liquid furniture polish) are chiefly aspiration hazards, with high potential to cause aspiration pneumonitis but rarely cause systemic symptoms.

Acute systemic toxicity: Acute systemic toxicity following hydro-carbon exposure is most commonly seen with aromatic and halogenated hydrocarbons and is primarily determined by their volatility and/or degree of absorption from the gastrointestinal tract. Acute effects include central nervous system (CNS) depression (e.g., lethargy, coma, and inebriation), seizures, and/or ventricular arrhythmias caused by myocardial sensitization to the endogenous and administered catecholamines (e.g., epinephrine). Besides, hepatic and acute renal tubular necrosis has been described after poisoning with the halogenated hydrocarbons, especially carbon tetrachloride (Gummin and Hryhorczuk, 2006).

Gases such as methane, ethane, propane, and butane cause asphyxia by replacing alveolar gas and are readily absorbed into the circulatory system. After absorption, they also have the potential to cause CNS depression, seizures, and ventricular arrhyth- 
mias due to myocardial sensitization to the catecholamines.

Other hydrocarbons have specific CNS effects after ingestion including the CNS depression (pine oil, gasoline, naphtha) and seizures (camphor). Systemic absorption is not a major contributor to pulmonary injury except in the setting of massive ingestion (Khine et al, 2009).

Pathophysiology: The hydrocarbon aspiration impacts the respiratory and central nervous systems. The respiratory system is primarily affected by direct injury. Low viscosity, low surface tension, and solvent properties of aspirated hydrocarbons with determine a compound's ability to cause chemical pneumonitis. The primary pathologic is severe necrotizing pneumonia.

Others are direct destruction of the airway epithelium, alveolar septae, and pulmonary capillaries, as well as solubilization of lipid surfactant layer. The secondary changes include atelectasis, interstitial inflammation, and hyaline membrane formation. The inflammatory response from chemical irritation generally causes temperature elevation, usually within hours of exposure (Lewander and Aleguas, 2007).

Volatile hydrocarbons are highly lipid soluble. They enter the circulation through the lungs and rapidly diffuse throughout the body and into the CNS (Dinwiddie, 1994). Neurons, which have high lipid content, are particularly susceptible to solvent properties. Manifestations in CNS secondary to severe pulmonary injury and hypoxia occurred (Gurwitz et al, 1978).

Clinical manifestations: Initial picture and diagnosis: Most patients who ingest aliphatic hydrocarbons (e.g., kerosene or pine oil) are children who are initially asymptomatic and have a history of exposure. Those patients with symptoms (e.g., respiratory distress, hypoxemia) soon after ingestion typically progress rapidly to respiratory failure.

When history of hydrocarbon exposure is lacking, characteristic odors can identify the presence and type of hydrocarbons ingested: Kerosene and other aliphatic hydrocarbons, Petroleum distillate odor Pine oil, Pine scent Halogenated hydrocarbons and Sweet solvent odor

Although exposure to some hydrocarbons may be confirmed by detection of urinary metabolites (e.g., tricholorethanol after chlorinated hydrocarbon exposure, hippuric acid after toluene exposure) or directly measured in the blood (e.g., toluene), these laboratory studies are not rapidly available and do not change management priorities. So, the diagnosis of hydrocarbon exposure is based on clinical features.

Physical findings post-hydrocarbon exposure: 1- Vital signs: Between 30 and $60 \%$ of patients with hydrocarbon aspiration have fever at the time of presentation $\left(38\right.$ to $40^{\circ} \mathrm{C}$ (100.4- $\left.104^{\circ} \mathrm{F}\right)$. Persistence beyond 48 hours suggests bacterial superinfection. The pulse oximetry may show decreased oxygen saturation (Ellenhorn, 1997).

2- Respiratory: Pulmonary manifestations secondary to aspiration generally occur within 30 minutes, although their onset may be delayed for 12 to 24 hours. Immediate signs of aspiration include coughing, choking, gagging, and vomiting. Respiratory examination findings vary with the degree of pulmonary injury and may include tachypnea, dyspnea, cyanosis, wheezing, diminished resonance on percussion, suppressed or tubular breath sounds, rales, nasal flaring, and/or grunting respirations. Displacement of alveolar gas by vaporized hydrocarbon may aggravate hypoxemia caused by inflammation and edema (Ellenhorn, 1997). The major pulmonary complications of hydrocarbon aspiration include asphyxia, necrotizing chemical pneumonitis, lipoid pneumonia, and hemorrhagic pulmonary edema, which quickly progresses to shock and respiratory arrest. Pneumothorax, subcutaneous emphysema of the chest wall, and pleural effusion, including empyema, may also occur. Secondary bacterial or viral infection may exacerbate the chemical 
pneumonitis. Pneumatoceles rarely develop in areas of extensive consolidation during the recovery period (Thalhammer et al, 2005).

3- Central nervous system: Hydrocarbon ingestion or inhalation may rapidly have direct central nervous system (CNS) effects, including somnolence, headache, ataxia, dizziness, blurred vision, weakness, fatigue, lethargy, stupor, seizures, and coma, depending on the amount ingested. In addition, hypoxia caused by hydrocarbon aspiration may cause secondary CNS toxicity, including drowsiness, tremors, or seizures.

4- Cardiovascular: Cardiac dysrhythmias and myocardial dysfunction may occur after hydrocarbon ingestion or inhalation. Solvent hydrocarbons (e.g., halogenated hydrocarbons), in particular, are known to produce fatal ventricular arrhythmias because they sensitize the myocardium to endogenous and exogenous catecholamines (sudden sniffing death).

5- Gastrointestinal: Ingestion of aliphatic hydrocarbons causes direct local irritation to the pharynx, esophagus, stomach, and small intestine, with edema and mucosal ulceration. Orogastric and intestinal irritation may be associated with nausea and hematemesis. These effects usually are mild and rarely require treatment (Wolfsdorf and Paed, 1976).

6- Liver and Kidney: Halogenated hydrocarbons may cause hepatic and/or renal tubular necrosis a day or two after ingestion.

7- Hematologic: Leukocytosis occurs early in the clinical course of hydrocarbon aspiration unrelated to pneumonitis and may last as long as one week. Hemolysis, hemoglobinuria, and consumptive coagulopathy rarely occur after large ingestions (Banner and Walson, 1983).

8- Ancillary studies: Children who ingest hydrocarbons should receive a chest radiograph four to six hours after exposure or sooner if they show signs of pulmonary aspiration.
Symptomatic patients should also undergo measurement of the following: Blood gas, complete blood count, serum glucose, serum electrolytes, and urinalysis. Besides, children exposed to halogenated hydrocarbons should have measures of the liver injury (aspartate aminotransferase (AST) and alanine aminotransferase (ALT)) and renal function (blood urea nitrogen and serum creatinine) performed.

The initial blood gas after hydrocarbon aspiration typically showed a mild respiretory alkalosis with hypoxemia. If hypoxemia is not corrected, the patient will develop a metabolic acidosis.

Radiographic findings: In patients who ultimately manifest the pulmonary aspiration post-hydrocarbon ingestion, the radiographic findings of hydrocarbon aspiration are evident within two hours of exposure in $88 \%$ and by 12 hours in $98 \%$. These findings frequently precede development of physical findings.

The initial radiograph findings consist of multiple, small, patchy densities with illdefined margins. The lesions become larger and coalesce as the injury progresses (Anas et al, 1981). In some cases, the radiographic findings may be minimal at a few hours and then rapidly progress to extensive infiltrates. Emphysema or pneumothorax may develop. Radiographic abnormalities typically peak between two and eight hours after the aspiration. The resolution of radiographic changes is gradual and lags behind clinical improvement, which usually occurs three to five days after aspiration. Pneumatoceles may develop during this latent period.

Differential Diagnosis: A clinician should consider the following alternative diagnoses when hydrocarbon exposure is not certain or is associated with findings suggestive of other poisonings: The bronchopneumoniaPatients with bronchopneumonia typically have fever and tachypnea and may appear similar to patients with the hydrocarbon aspiration. Nevertheless, the patients with pneumonia do not display a characteristic 
petroleum distillate odor and do not have findings of chemical pneumonitis on chest radiograph. The salicylate overdose-Patients who have ingested salicylates may have fever and tachypnea with altered mental status but typically do not have abnormal chest radiographs soon after ingestion. Some clinicians may think the fruity odor of ketoacidosis is similar to that caused by halogenated hydrocarbon ingestion. Other toxins- Any toxin that produces metabolic acidosis (e.g., iron, metformin, isoniazid, toxic alcohol) or hypoxia (e.g., carbon monoxide, cyanide) may cause tachypnea. Blood gas measurement often differentiate these toxins from hydrocarbon exposure with its typical the respiratory alkalosis.

Hydrocarbons are sometimes used as solvents for other dangerous toxins (e.g., organophosphates). So, the clinician should seek to identify the exact compound that was ingested when managing a patient with hydrocarbon poisoning to ensure timely the recognition and treatment of all possible toxic agents.

Resources: The regional poison control centers in the United States are available at all times for consultation on patients who are critically ill, require admission, or have clinical pictures that are unclear (1-800-2221222). Besides, some hospitals have clinical and/or medical toxicologists available for bedside consultation and/or inpatient care. Whenever available, these are invaluable resources to help in the diagnosis and management of ingestions or overdoses. The WHO provides a listing of international poison centers at its website: www.who.int/ ipcs/poisons/centre/directory/en.

Management: Successful management of hydrocarbon aspiration requires recognition of pulmonary toxicity and rapid initiation of appropriate supportive care.

Stabilization: Initial treatment depends on the degree of immediate toxicity including the presence of respiratory distress and altered mental status: Patients with the severe respiratory distress, unresponsive to oxygen and beta- 2 bronchodilators (e.g., albuterol) or significant altered mental status (lethargy, coma) shortly after hydrocarbon ingestion should undergo endotracheal intubation. The patients with seizures should receive the intravenous benzodiazepine therapy (e.g., lorazepam $0.1 \mathrm{mg} / \mathrm{kg}$ ) in addition to support of airway and breathing. Patients with mild to moderate respiratory symptoms on presentation are at risk for the development of significant cemical pneumonitis with progression to respiratory failure over next 24 to 48 hours. In addition, to supplemental oxygen, as needed, and close monitoring, they should have chest radiographs performed at the presentation time. These patients should remain NPO (nothing by mouth) during the initial observation period and receive maintenance intravenous fluids. The patients who are asymptomatic at presentation warrant serial examinations with monitoring. Such patients should also remain NPO (nothing by mouth) during the initial observation period. The radiographic evaluation should occur at four to six hours after hydrocarbon ingestion or sooner if they become symptomatic or have additional pulmonary aspiration risk.

External decontamination: Prior to the initiating decontamination of patients with the external hydrocarbon exposure, the health care team should done appropriate personal protective equipment appropriate to the hazard posed by the specific chemical.

Contaminated clothing should be removed to prevent continued inhalation exposure. Affected skin and hair should be cleansed as many hydrocarbons cause irritation. Certain hydrocarbon compounds (e.g., gasoline, methylene chloride) cause cell membrane injury and dissolution of lipids, which results in skin necrosis and burns with prolonged exposure. Treatment of dermal and ocular exposure initially consists of copious water irrigation (Arena, 1987).

Gastrointestinal decontamination: For the patients who have ingested a hydrocarbon, it is recommended not performing either syrup 
of ipecac administration or gastric lavage with a large bore orogastric tube. In these patients, the risk of additional aspiration outweighs the potential benefit obtained from these procedures. Some toxicologists suggest nasogastric lavage for the removal of hydrocarbons with significant systemic toxicity in the rare patient who has ingested a large amount (e.g., suicidal ingestion of trichloro-ethylene) and who presents within one hour of ingestion (Shannon, 2000).

This recommendation is based on the randomized controlled trials in poisoned patients that evaluated the use of syrup of ipecac or gastric lavage with a large bore orogastric tube. These studies found no definite clinical benefit from induced vomiting or gastric lavage after any ingestion. Besides, both of these procedures may increase the aspiration risk, especially after hydrocarbon ingestion (Krenzelok et al, 1997).

Activated charcoal (AC) should not be administered to patients with a single agent ingestion of a hydrocarbon without systemic toxicity for the following reasons: $\mathrm{AC}$ administration increases the risk of spontaneous vomiting and additional pulmonary aspiration. AC does not bind well to hydrocarbons. In this instance, toxicity arises from pulmonary aspiration rather than systemic toxin absorption.

Systemic toxicity: Consultation with a regional poison control center is strongly advised when determining the need to provide gastro-intestinal decontamination procedures in patients who have ingested hydrocarbons.

For patients who have ingested large quantities of hydrocarbons with significant potential for systemic toxicity and who present for care within 60 minutes of ingestion, we suggest gastric emptying using nasogastric lavage. These agents can be remembered by using the mnemonic "CHAMP": C: Camphor H: Halogenated hydrocarbons (e.g., trichloroethane, trichloroethylene) A: Aromatic hydrocarbons (e.g., benzene, toluene, xylene) M: Metal additives (e.g., arsenic, mercury) in hydrocarbon base P: Pesticide additives (e.g., organophosphates) in hydrocarbon base.

Some experts also suggest nasogastric lavage in patients who ingest large amounts of aliphatic hydrocarbons (e.g., suicide attempt in an adult patient) because of the potential for systemic absorption with the central nervous system and pulmonary toxicity.

This recommendation is based on clinical experience regarding how to manage this uncommon scenario and randomized trials that indicate little benefit of gastric lavage more than 60 minutes after ingestion. The nasogastric lavage should only be done when the risk of systemic toxicity from gastrointestinal absorption of hydrocarbon is equal to or exceeds the increased risk of pulmonary aspiration associated with the nasogastric lavage.

Nasogastric lavage should be withheld in patients with a depressed mental status who may not be able to protect their airway, unless endotracheal intubation is performed first. The following measures, performed before nasogastric lavage, may minimize the risk of aspiration: Endotracheal intubation with a balloon-cuffed endotracheal tube Left lateral decubitus with the Trendelenburg positioning Pinching off the nasogastric tube and withdrawing it quickly after procedure is complete (Vale, 1997).

The patients who ingest hydrocarbons that are combined with an adsorbable toxic additive (e.g., organophosphates) or who congest other bind toxic substances (e.g., prescription medications) receive activated charcoal ((Lewander and Aleguas, 2007).

The recommendation of $\mathrm{AC}$ administration in this select group of patients who ingest hydrocarbons derives from indirect evidence of benefit in volunteers, animal studies, and evidence of benefit following 
ingestions of other substances. Because of adverse effects, such as vomiting and dehydration, the combination of a cathartic (e.g., sorbitol) and AC should be used sparingly, if at all and only a single dose of a cathartic should be given to any patient (Vale and Kulig, 2004).

Pulmonary management: The treatment of hydrocarbon pneumonitis is supportive and includes oxygen and close monitoring of respiratory status.

Bronchospasm should be treated with the selective beta- 2 agonists. The epinephrine and isoproterenol should be avoided because they can cause fatal ventricular dysrhythmia in the hydrocarbon-sensitized myocardium (Chyka and Seger, 1997).

Endotracheal intubation and conventional mechanical ventilation is indicated in patients with respiratory failure or in situations where mental status makes them unable to maintain their airway (Zucker et al, 1986).

Extracorporeal membrane oxygenation (ECMO) or the high frequency ventilation are therapeutic options for the small minority of children who have hydrocarbon pneumonitis and respiratory failure with the severe hypoxemia unresponsive to conventional mechanical ventilation. The value of these interventions in adults is not known. However, children are more likely to have the ability to regenerate new lung tissue after injury (Bysani et al, 1994).

In a case series that evaluated survival after ECMO, 13 of 19 children $(68 \%)$ with the hydro-carbon pneumonitis survived versus 459 of 883 children $(52 \%)$ who had other respiratory disease. This experience suggests that the pulmonary injury sustained by children with hydrocarbon pneumonitis is reversible in some patients and that ECMO may be life-saving in selected patients with the severe hydrocarbon pneumonitis unresponsive to the conventional mechanical ventilation (Chyka, 1996).

Case reports also indicate that high frequency ventilation (e.g., jet ventilation, high frequency oscillatory ventilation, or high frequency percussion ventilation) may be life-saving procedures for children with severe hydrocarbon pneumonitis unresponsive to conventional mechanical ventilation (Mabe et al, 2007).

Corticosteroids have shown no beneficial effect on the course of the hydrocarbon aspiration in observational studies and may be harmful (Lee et al, 2003).

Pneumonitis caused by hydrocarbon aspiration should not be treated routinely with antibiotics unless signs of secondary infection, including the following, are present: Recurrence of fever after first 48 hours. Increasing infiltrate in chest radiograph Leukocytosis after the first 48 hours Sputum or tracheal aspirate proved positive for bacteria (Karlson, 1982). Pneumatoceles usually resolve spontaneously and do not require specific treatment.

Disposition: Clinical findings and radiographic results largely determine patient disposition after hydrocarbon ingestion. Indications for immediate admission of patients who have ingested or aspirated hydrocarbons include (Shannon, 1991). Symptomatic patient, the patient with suicidal intent or massive ingestion indications for admission that become apparent up to six hours after hydrocarbon ingestion include: Patient with a normal or mildly abnormal initial chest radiograph who develops symptoms during the observation period Patient who develops symptoms related to toxic additives during the observation period (e.g., heavy metal or organophosphate insecticide) Patient with mild symptoms and normal chest radiograph who fails to improve during the observation period. All patients for who close follow-up cannot be established. Indications for discharge after six hours of observation include: Asymptomatic patients with normal chest radiograph obtained four or more hours post exposure. The asymptomatic patients with mildly abnormal chest radiographs who do not develop symptoms 
during the observation period can receive Prognosis: Although most children survive without complications or sequelae, some progress rapidly to respiratory failure and death. The central nervous system effects (e.g., somnolence, convulsions, and coma) and/or pulmonary symptoms may dominate course. Prognosis is affected by the volume of ingestion or aspiration, the specific agent involved, and the adequacy of medical care. The typical clinical course averages two to five days. Mild CNS depression soon after ingestion seldom produces serious morbidity provided that pulmonary involvement does not occur (Klein and Simon, 1986). The small airways are at greatest risk for longterm injury. One study examined pulmonary function in 17 children 8 to 14 years after hydrocarbon aspiration. More than $80 \%$ had at least one pulmonary function abnormality. Clinical significance is uncertain (David et al, 2013).

Recommendations: Clinical features of hydrocarbons are a diverse group of organic carbon compounds that cause respiratory distress and chemical pneumonitis after ingestion as a result of the indoor spraying of insecticide pulmonary aspiration. The physical findings of pulmonary aspiration may be delayed until several hours after ingestion. Acute systemic toxicity varies by specific agent ingested. Potential systemic toxicity includes the central nervous system depression, the seizures, and the cardiac arrhythmias. Decontamination Patients with external hydrocarbon exposure should have contaminated clothing removed. Treatment of dermal and ocular exposure consists of copious water irrigation. Prior to initiating decontamination of patients with external hydrocarbon exposure, the health care team should don appropriate personal protective equipment appropriate to the hazard posed by the specific chemical. For patients who have ingested a hydrocarbon, it is not recommended to perform gastric emptying procedures with the syrup of ipecac administration or gastric lavage with a large timely outpatient follow-up the next day. bore orogastric tube (Grade 1B). Activated charcoal (AC) should not be administered to patients with a single agent ingestion of a hydrocarbon without systemic toxicity. For patients who have ingested a large amount of hydrocarbons with significant potential for systemic toxicity and who present for care within 60 minutes of ingestion, it was suggested that gastric emptying using nasogastric lavage (Grade 2C). It was suggested patients who ingest hydrocarbons that are combined with an adsorbable toxic additive (e.g., organophosphates) or who congests other toxic substances that are adsorbable (e.g., prescription medications) receive oral activated charcoal $(1 \mathrm{~g} / \mathrm{kg})$ (Grade 2C).

Treatment of the hydrocarbon poisoning is primarily supportive and involves close assessment of respiratory status, including the chest radiograph. Specific interventions include supplemental oxygen, inhaled albuterol for bronchospasm, and initiation of NPO (nothing by mouth) status, and endotracheal intubation for patients with signs of respiratory failure. Administration of exogenous catecholamines (e.g., epinephrine), prophylactic antibiotics, or corticesteroids should be avoided. Clinical findings and radiographic results largely define patient disposition after hydrocarbon ingestion.

In Egypt: Many authors dealt with hydrocarbon hazards, the following were the most interesting ones from the medical of view.

Soliman et al. (1997) evaluated serum organochlorine levels among 31 Egyptian patients suffering from colorectal and 17 controls. The highest levels and large interindividual variability of the $p, p$ '-dichlorodiphenyldicholoroloro ethylene (DDE), dichlorodiphenyl-trichloroanthane(DDT), betahexachlorocyclohexane (beta- $\mathrm{HCH}$ ), and hexachlorobenzene (HCB) levels were found among most subjects, especially those from the rural areas. Farming and aging were each associated positively with high serum organochlorines. The colorectal 
cancer patients had higher serum organochlorines levels than the controls, high levels of organochlorines related to age, residence, occupation, and colorectal cancer in a larger population in Egypt.

Morsy et al. (1998) stated that nowadays, there are many problems with the using of chemical insecticides as resistance, environmental pollution, toxicity to man and animal...etc, which required the investigation of a new line for controlling arthropod pests of medical and agricultural importance.

Jaga (1999) in Egypt reported sera organochlorine pesticide levels in patients with colorectal cancer. Ahmed et al. (2002) in Port Said studied residues of 1,1-dichloro2,2-bis(p-chlorophenyl) ethylene (DDE) and polychlorinated biphenyls (PCBs) in the blood serum samples collected from a cohort of 43 fasting females diagnosed with the invasive adenocarcinoma of the breast, 21 female suffering benign breast disease, and 11 normal healthy females attending the health insurance outpatient clinicbetween July 1999 and July 2000.. Mean residues of DDE detected in the three examined groups of females were 41+/-5.2, 48+/-6.2 and $31+/-2.5 \mathrm{ng} / \mathrm{g}$ for breast cancer cases, benign breast disease cases \& controls, respectively indicating some significantly less residues in blood serum of control females. While PCBs residues detected were $54+/-17,59+/-23$ and $61+/-21 \mathrm{ng} / \mathrm{g}$, for the three, respectively. Soliman et al. (2003) assessed determinants of the organochlorine serum levels among the premenopausal 69 breast cancer patients and 53 controls consisting of visitors to the hospitals of the cancer patient's women and the risk of premenopausal breast cancer for women with high organochlorine serum levels. Mean DDE levels were 12.7+/-20.3 $\mathrm{ppb}$ for cases and 16.6+/-30.1 ppb for controls $(\mathrm{P}=0.60)$; beta-HCH levels were 2.1 $+/-3.8 \mathrm{ppb}$ for patients and 2.1+/-3.9 ppb for controls $(\mathrm{P}=0.71)$. Interestingly, subjects with low levels had breast fed their children for an average period of 18 months. Women with no lactation history had much higher organochlorine levels than women who breast fed ( $\mathrm{P}=0.002$ for DDE). Younger age, older age at first childbirth and shorter duration of breast feeding were significant predictors of higher levels of serum DDE levels. Younger age, older age at first childbirth, and higher body mass index were significant predictors of higher beta- $\mathrm{HCH}$ levels. They suggested that organochlorine serum level was not a risk factor of breast cancer in this population. El Nemr and AbdAllah (2004) analyzed ten organo-chlorine compounds in four species of fish (Bouri Mugil spp., Denis Sparus auratus, Moza Boops boops and Mousa Pegusa lascaris), from four costal governorates South Sinai, Suez, Port-Said and Damietta. The grand total values recorded were in descending order: Bouri $>$ Denis $>$ Moza $>$ Mousa. The highest concentration of pesticides was recorded in Damietta fish $(20-211 \mathrm{ng} / \mathrm{g}$ of wet weight) and the p,p'-DDE dominated over the other $\mathrm{p}, \mathrm{p}^{\prime}$-isomers in all fish, while the dieldrin was dominated in the studied cyclodiene compounds. Nevertheless, the hexachlorobenzene (HCB) was at all studied governorate fish except Mousa fish from South Sinai. Toxaphene was detected only in fish (Bouri, Denis, and Mousa) from Demietta governorate. Chlordane was not detected in any studied fish. The level of organochlorine pesticides contamination in fish from the studied governorates is relatively low and should not pose a health risk to consumers.

Malki et al. (2009) stated that garlicderived organosulfur compounds (OSCs) have highly effective antitumor effects, but the mechanism has yet to be investigated. Garlic constituent diallyl trisulfide (DATS) suppresses viability of cultured MCF-7 and MCF-12a cells respectively by decreasing the percent of cells in $\mathrm{G}(2) / \mathrm{M}$ and inducing apoptotic cell death. The DATS-induced apoptosis was markedly elevated in MCF-7 cells compared with MCF-12a cells and this was correlated with elevated levels of cyclin B1. The semi-quantitative and real-time RT- 
PCR indicated that DATS-enhanced the expression levels of FAS and cyclin D1, but in contrast, down regulated the expression levels of Akt and Bcl-2. The DAT-induce apoptosis was correlated with the induction of proapoptotic Bax protein and p53 protein expression was upregulated and translocation to the nucleus in MCF-7 cells. They concluded that the DATS application might offer the novel strategy for treatment of the human breast cancer. Malhat et al. (2012) estimated Etofenprox residues by standardized QuEChERS technique in the tomato following an application of Trebon ${ }^{\circledR} 20 \%$ EC. The average recoveries of etofenprox on tomato for fortification levels $0.01,0.1$ and $0.5 \mathrm{mg} / \mathrm{kg}$ were $87.5 \%, 89.7 \%$ and $92.2 \%$, respectively, with relative standard division of 3.50, 4.11 and 3.20. The LOQ for tomato was found to be $0.01 \mathrm{mg} / \mathrm{kg}$. The average initial etofenprox deposit on tomato was $0.783 \mathrm{mg} / \mathrm{kg}$, at one application rate. This etofenprox residue dissipated below its LOQ of 0.01 after 15 days at a single dosage. Half-life of the etofenprox was detected 2.15 days, at the recommended dosage. El-Demerdash (2011a) studied the effects of the organophosphorus [fenitrothion (FNT)] and pyrethroid (lambdacyhalothrin (LC)] pesticides mixture on the antioxidant defense system and liver function enzymes in rat liver. He found that FNT+LC caused a significant concentration-dependent \& time-related inhibition of glutathione S-transferase (GST), superoxide dismutase (SOD) \& catalase (CAT) activities, and reduction of glutathione (GSH) content. The depletion of GSH might indicate that reactive oxygen species could be involved in the toxic effects of FNT + LC. Also, a significant inhibition in transaminases (AST, ALT) and phosphatases (AcP, AlP) activities was observed. On the other hand, FNT+LC increased activity of lactate dehydrogenase (LDH) and thiobarbituric acid reactive substances (TBARS) levels. Khairy et al. (2012) in Abo-Qir Bay (Eastern of Alexandria Governorate), assessed presence and distribution of polychlori- nated biphenyls (PCBs), organochlorine, and chlorpyrifos in the sediments and mussels and their risks. They added that by a SLERA, it was possible to indicate which class of chlorinated organic compounds was of highest concern to assess and to improve environmental quality of the bay. The monitoring of organochlorines and chlorpyrifos would be needed to control the future trend of pollution. El-Demerdash (2011b) studied the effect of a broad spectrum insecticide, composed of a mixture of organophosphate plus pyrethroids (fenitrothion 25\%, lambda cyhalothrin $2.5 \%$ and piperonyl butoxide $6 \%$, on antioxidant status and oxidative stress biomarkers in rat brain. Exposure to insecticide mixture resulted in a significant increase in thiobarbituric acid reactive substances, associated with decreased level of reduced glutathione (GSH), superoxide dismutase (SOD), catalase (CAT), glutathione S-transferase (GST) and acetylcholinesterase activities and beside protein content in rat brain. However, a significant induction of lactate dehydrogenase (LDH) activities was observed. The response was concentration and time dependent. Results showed that the used insecticides had the propensity to cause significant oxidative damage in rat brain, associated with marked perturbations in antioxidant defense system in addition to antioxidant enzymes can be used as potential biomarkers of toxicity associated with pesticides exposure. Hussien et al. (2011) found that cypermethrin increased thiobarbituric acid-reactive substances, and decreased glutathione and activities of antioxidant enzymes. Brain showed damage, reduction in activities of acetylcholineesterase and monoamine oxidese, total protein, albumin, body weight, induction in triacylglycerol and cholesterol. Sesame oil treatment gave protection against cypermethrin-induced brain toxicity of female rats and all haemtological changes returned to normal levels.

El-Bahnawasy et al. (2012) stated that human lice infestation (pediculosis) is very common in hundreds of millions worldwide 
including Egypt. People who live and work in close proximity to louse-infested areas may acquire lice even if they regularly wash their clothes and have good hygiene. All louse-infested persons and workers in close contact with louse-infested persons should periodically inspected and use long-acting safe insecticides. They added that lice can be treated with agents such as DDT, malathion, and Lindane, but reports of resistance to one or more of them have recently appeared. Pyrethroid permethrin when applied as a dust or spray to clothing or bedding was highly effective against lice and was the delousing agent of choice.

\section{Conclusion}

Sometimes hydrocarbon insecticides are a must. However, their miss-use is connected at least with the high incidence of colorectal cancer in Egyptian children and youth. Most of environmental exposures are involved to several pesticide agents in air, water, and food. The use of genotoxicity testing proved essential for assessment of potential human toxicity to prevent hazards.

The cytogenetic monitoring of human populations exposed to the environmental agents has proved to be a useful tool to detect mutagenic effects. The cytogenetic analysis of human chromosomes in the peripheral lymphocytes allows direct detection of mutation in somatic cells.

\section{References}

Ahmed, MT, Loutfy, N, El Shiekh, E, 2002: Residue levels of DDE and PCBs in the blood serum of women in the Port Said region of Egypt. J. Hazard Mater. 89, 1:41-8.

Anas, N, Namasonth, V, Ginsburg, CM, 1981: Criteria for hospitalizing children who have ingested products containing hydrocarbons. JAMA 246:840.

Arena, JM, 1987: Hydrocarbon poisoning-current management. Pediatr. Ann. 16: 879-84.

Banner, WJr, Walson, PD, 1983: Systemic toxicity following gasoline aspiration. Am. J. Emerg. Med.1:292-8.

Barone, S, 2002: Child-resistant packaging. Consumer product safety review:6:3.www. cpsc. gov/cpscpub/pubs/cpsr_nws23.pdf
Bronstein, AC, Spyker, DA, Cantilena, L RJr, et al. 2007: Annual report of the American Association of Poison Control Centers' National Poison Data System (NPDS). Clin. Toxicol. (Phila) 45:815-20.

Bronstein, AC, Spyker, DA, Cantilena, L RJr, et al. 2008: Annual report of the American Association of Poison Control Centers' National Poison Data System (NPDS): $25^{\text {th }}$ Ann. Rept. Clin. Toxicol. (Phila) 46:927-32.

Bysani, GK, Rucoba, RJ, Noah, ZL, 1994: Treatment of hydrocarbon pneumonitis: High frequency jet ventilation as an alternative to extracorporeal membrane oxygenation. Chest 106:300-9.

Chyka, PA, 1996: Benefits of extra-corporeal membrane oxygenation for hydrocarbon pneumonitis. J. Toxicol. Clin. Toxicol. 34:357-64.

Chyka, PA, Seger, D, 1997: Position statement: Single-dose activated charcoal: American Academy of Clinical Toxicology; European Association of Poisons Centres and Clinical Toxicologists. J. Toxicol. Clin. Toxicol. 35:721-9.

David, JP, Ismail, HM, Chandor-Proust, A, Paine, MJ, 2013: Role of cytochrome P450s in insecticide resistance: Impact on the control of mosquito-borne diseases and use of insecticides on Earth. Philos Trans. R. Soc. Lond. B Biol. Sci. 368(1612):20120429.

Dinwiddie, SH, 1994: Abuse of inhalants: A review. Addiction 89:925-31.

El-Bahnasawy, MM, Abdel Fadeel, EE, Morsy TA, 2012: Human pediculosis: A critical health problem and what about nursing policy? J. Egypt. Soc. Parasitol. 42, 3:541-62.

El-Demerdash, FM, 2011a: Oxidative stress and hepatotoxicity induced by synthetic pyrethroids-organophosphate insecticides mixture in rat. J. Environ. Sci. Hlth. C Environ. Carcinog. Ecoto-xicol. Rev. 29, 2:145-58

El-Demerdash, FM, 2011b: Lipid peroxidation, oxidative stress and acetylcholinesterase in rat brain exposed to organophosphate and pyrethroid insecticides. Food Chem. Toxicol. 49, 6: 1346-52.

Ellenhorn, MJ, 1997: The hydrocarbon products. In: Ellenhorn's Medical Toxicology: Diagnosis and Treatment of Human Poisoning, $2^{\text {nd }}$ ed, Ellen, MJ, Schonwald, S, Ordog, G, Wasserberger (Eds), Williams and Wilkins, Baltimore.

El Nemr, A, Abd-Allah, AM, 2004: Organochlorine contamination in some marketable fish in Egypt. Chemosphere 54, 10:1401-6. 
Gummin, DD, Hryhorczuk, DO, 2006: Hydrocarbons. In: Goldfrank's Toxicologic Emergencies, $8^{\text {th }}$ ed, Flomenbaum, NE, Goldfrank, LR, Hoffman, RS, et al (eds), McGraw-Hill, N. Y.

Gurwitz, D, Kattan, M, Levison, H, Culham, JA, 1978: Pulmonary function abnormalities in asymptomatic children after hydrocarbon pneumonitis. Pediatrics 62:789-94

Hussien, HM, Abdou, HM, Yousef, MI, 2011: Cypermethrin induced damage in genomic DNA and histopathological changes in brain and haematotoxicity in rats: the protective effect of sesame oil. Brain Res. Bull. 92:76-83.

Jaga, K, 1999: Serum organochlorine pesticide levels in patients with colorectal can-cer in Egypt. Arch. Environ. Hlth. 54, 3:217-8.

Karlson, KH Jr, 1982: Hydrocarbon poisoning in children. South Med. J. 75:839-44.

Khairy, MA, Kolb, M, Mostafa, AR, El-Fiky, A, Bahadir, M, 2012: Risk posed by chlorineted organic compounds in Abu Qir Bay, East Alexandria, Egypt. Environ. Sci. Pollut. Res. Int. 19, 3:794-811.

Khambay, BPS, Jewess, PJ, 2004: Pyrethroids. In: Gilbert, LI, Iatrou, K, Gill, SS, eds. Comprehensive Molecular Insect Science. Vol. 6. Oxford: Pergamon Press.

Khine, H, Weiss, D, Graber, N, et al, 2009: A cluster of children with seizures caused by camphor poisoning. Pediatrics 123:1269-74.

Klein, BL, Simon, JE, 1986: Hydrocarbon poisonings. Pediatr. Clin. North Am. 33:411-20.

Krenzelok, EP, McGuigan, M, Lheur, P, 1997: Position statement: ipecac syrup: American Academy of Clinical Toxicology; European Association of Poisons Centres and Clinical Toxicologists. J. Toxicol. Clin. Toxicol. 35:699714.

Lee, LK, Shannon, MS, Arnold, JH, 2003: The use of high frequency oscillatory ventilation in hydrocarbon pneumonitis. Int. J. Med. Toxicol. 6:10-9.

Lewander, WJ, Aleguas, A, 2007: Petroleum distillates and plant hydrocarbons. In: Haddad and Winchester's Clinical Management of the Poisoning and Drug Overdose, $4^{\text {th }}$ ed, Shannon, MW, Borron, SW, Burns, MJ (Eds), Saunders Elsevier, Philadelphia.

Mabe, TG, Honeycutt, T, Cairns, BA, et al, 2007: High-frequency percussive ventilation in a pediatric patient with hydrocarbon aspiration. Pediatr. Crit. Care Med. 8:383-90.
Malhat, F, Abdallah, H, Nasr, I, 2012: Estimation of etofenprox residues in tomato fruits by QuEChERS methodology and HPLC-DAD. Bull. Environ. Contam. Toxicol. 88, 6:891-3

Malki, A, El-Saadani, M, Sultan, AS, 2009: Garlic constituent diallyl trisulfide induced apoptosis in MCF7 human breast cancer cells. Cancer Biol. Ther. 8, 22:2175-85.

Morsy, TA, Shoukry, A, Mazyad, SA, Makled, KM, 1998: The effect of the volatile oils of Chenopodium ambrosioides and Thymus vulgaris against the larvae of Lucilia sericata (Meigen). J. Egypt. Soc. Parasitol. 28, 2:503-10.

Shannon, M, 1991: Petroleum Distillate Poisoning. Harwood-Nuss, A, JP Lippincott.

Shannon, M, 2000: Ingestion of toxic substances by children. N. Engl. J. Med. 342:186-94. Soliman, AS, Smith, MA, Cooper, SP, Ismail, $\mathrm{K}$, Khaled, H, et al, 1997: Serum organochlorine pesticide levels in patients with colorectal cancer in Egypt. Arch. Environ. Hlth. 52, 6:40915.

Soliman, AS, Wang, X, DiGiovanni, J, Eissa, S, Morad, M, et al, 2003: Serum organochlorine levels and history of lactation in Egypt. Environ. Res. 92, 2:110-7.

Thalhammer, GH, Eber, E, Zach, MS, 2005: Pneumonitis and pneumatoceles following accidental hydrocarbon aspiration in children. Wien Klin. Wochenschr. 117:150-60.

Vale, JA, 1997: Position statement: gastric lavage: American Academy of Clinical Toxicology; European Association of Poisons Centres and Clinical Toxicologists. J. Toxicol. Clin. Toxicol. 35:711-9.

Vale, JA, Kulig, K, 2004: American Aca-demy of Clinical Toxicology; European Association of Poisons Centres and Clinical Toxicologists. Position paper: gastric lavage. J. Toxicol. Clin. Toxicol 42:933-40.

Van Gorcum, TF, Hunault, CC, Van Zoe-len, GA, et al, 2009: Lamp oil poisoning: did the European guideline reduce the number and severity of intoxications? Clin Toxicol (Phila) 47:29-34.

WHO, 2010: World Malaria Report. Geneva.

Wolfsdorf, J, Paed, D, 1976: Kerosene intoxication: an experimental approach to the etiology of the CNS manifestations in primates. J. Pediatr. 88:1037-42.

Zucker, AR, Berger, S, Wood, LD, 1986: Management of kerosene-induced pulmonary injury. Crit. Care Med. 14:303-8. 